\title{
ESOFAGECTOMÍA:
}

\section{EXPERIENCIA DE DIEZ AÑOS EN EL \\ HOSPITAL DE SAN JOSÉ. 1991-2001}

Andrés Díaz Ramírez, MD. , Juan Camilo Ramírez, MD.**

\section{Resumen}

El presente estudio revisa la experiencia durante diez años en el Hospital de San José, en pacientes que fueron sometidos a esofagectomía transhiatal o transtorácica, por enfermedad tanto maligna como benigna. El propósito es comparar los resultados obtenidos en nuestra serie con otras más grandes reportadas en la literatura.

Se revisaron las historias clínicas de quienes fueron sometidos a los procedimientos mencionados, en el periodo de 1991 a 2001. Se incluyeron 17 pacientes, 16 hombres y una mujer; tres de ellos con lesiones benignas y 14 con enfermedad maligna, siendo la variante histológica más frecuente el adenocarcinoma. Los síntomas predominantes fueron disfagia y disminución de peso. Se realizaron 12 procedimientos transhiatales y tres transtorácicos, con $35 \%$ de complicaciones postoperatorias, entre ellas fístula de la anastomosis esofagogástrica, neumonía y arritmias. El sangrado intraoperatorio fue en promedio de $1200 \mathrm{~mL}$. Es infortunado que nuestro estudio no tuvo seguimiento alguno, de manera que es imposible la evaluación objetiva de los resultados a mediano y largo plazo. La muestra obtenida fue muy pequeña, por lo cual consideramos que se deben realizar nuevas investigaciones con una población mayor.

\section{Introduccilón}

A pesar de los avances recientes con otras modalidades terapéuticas, como la radioterapia y la quimioterapia, la cirugía todavía es la alternativa que brinda mayor posibilidad de curación en cáncer de esófago. Sin embargo, la tasa de sobrevida no se ha modificado de manera considerable en las últimas décadas.

Por años, el procedimiento de elección fue el abordaje transtorácico, en el cual se remueven el esófago y el tejido periesofágico junto con los ganglios linfáticos, gracias al abordaje combinado de una laparotomía posterolateral derecha con o sin cervicotomía.

Durante las dos últimas décadas algunos cirujanos, especialmente Orringer, promovieron e impulsaron el uso de otro tipo de abordaje para la resección del esófago, tanto para patología benigna como para tumores esofágicos malignos, en especial del tercio inferior. Este autor lo denominó esofagectomía transhiatal, con muy

\footnotetext{
Residente Médico Cirujano.

** Cirujano de tórax. Profesor Asociado de Cirugía General. Hospital de San José.
}

buenos resultados reportados en cuanto a sobrevida y disminución de la morbilidad, comparado con la vía transtorácica. Argumenta que los abordajes combinados en individuos de por sí debilitados por su patología tumoral, conducen a complicaciones postoperatorias, en especial pulmonares, además de aquellas potencialmente letales, como la fístula de la anastomosis intratorácica.

La tasa de mortalidad depende del estadío del tumor y de la condición general del paciente, aunque también de las complicaciones postoperatorias relacionadas con el procedimiento, como fístula de la anastomosis, mediastinitis, neumonía, arritmias e infarto agudo de miocardio.

El cáncer de esófago es uno de los retos mas grandes a los que se enfrenta el cirujano en su práctica, debido a la relación anatómica y la localización del tumor, que conduce a un tratamiento quirúrgico de magnitud y extensión considerables, con la conocida morbilidad asociada al procedimiento.

Como lo mencionan varios autores, "ningún paciente es más desdichado que aquel que sufre una obstruc- 
ción maligna del esófago, porque muere de lenta inanición" y "no se puede morir dignamente sin poder deglutir". Es por ello que la cirugía se convierte en el pilar del tratamiento de cáncer esofágico.

También se emplean las mismas técnicas para el manejo de algunas enfermedades benignas, como la quemadura esofágica por cáusticos, el megaesófago y las reoperaciones de pacientes con cirugías previas para corrección de reflujo gastroesofágico y síntomas recidivantes, en quienes no es posible llevar a cabo otro procedimiento reconstructivo.

\section{Materiales y métodos}

El presente es un estudio observacional descriptivo de serie de casos retrospectivo, que incluyó la revisión de las historias clínicas de todos los pacientes que fueron sometidos a esofagectomía transhiatal o transtorácica por carcinoma de esófago o por enfermedad benigna en el Hospital de San José, en el período comprendido entre 1991 y 2001.

Las variables investigadas fueron edad, sexo, manifestaciones clínicas, estado nutricional, antecedentes de tabaquismo y consumo de alcohol, enfermedades asociadas y localización de la lesión. Con respecto al procedimiento en sí, se consideraron la indicación (lesión benigna o maligna), vía de la resección (transhiatal o transtorácica), sangrado estimado, tipo de anastomosis (manual o mecánica, cervical o torácica) y tiempo de cirugía. En cuanto al estado postoperatorio de los pacientes, las variables incluyeron: número de días de hospitalización, en la unidad de cuidado intensivo y de ventilación mecánica, así como las complicaciones postoperatorias, la mortalidad perioperatoria y los resultados del seguimiento.

\section{Resultados}

Se estudiaron 17 pacientes, 16 hombres y una mujer, entre los 15 y 75 años, con promedio de 54,5 años. Ocho tenían antecedente de tabaquismo y cinco de consumo de alcohol importante durante su vida. Catorce tenían diagnóstico de enfermedad maligna, siendo el adenocarcinoma la variante histológica más frecuente con nueve casos, seguido por carcinoma escamolecular en cuatro y carcinoma mal diferenciado, presente en uno. Tres tenían enfermedad benigna, dos con quemadura del esófago por cáusticos y reoperación por acalasia en otro (Tabla $\mathbf{1}$ ).

La localización de los tumores fue el tercio distal en 12 de los casos, dos en el medio y ninguno en el superior. El síntoma predominante fue la disfagia, presente en 15 de los sujetos, seguida por dolor epigástrico en diez y pérdida de peso en once. Otras manifestaciones frecuentes fueron vómito, melenas, hematemesis y dolor retroesternal, cada una en dos pacientes. En uno de los participantes la pirosis fue la queja principal. La duración de los síntomas osciló entre un mes y tres años (Tabla 2).

Tres pacientes tenían condiciones médicas asociadas, uno hipertensión arterial, otro enfermedad pulmonar obstructiva crónica y un tercero enfermedad del nodo sinusal. La clasificación de ASA no se registró en gran parte de las historias revisadas.

\section{Tabla I. Indicación del procedimiento}

\begin{tabular}{ll} 
Patologia & Número y porcentaje \\
\hline Maligna & 14 \\
Adenocarcinoma & $9(52,9 \%)$ \\
Carcinoma escamocelular & $4(23,5 \%)$ \\
Carcinoma indiferenciado & $1(5,8 \%)$ \\
Benigna & 3 \\
Quemadura por cáusticos & $2(11,7 \%)$ \\
Acalasia & $1(5,8 \%)$
\end{tabular}

Tabla 2. Síntomas predominantes

\begin{tabular}{lcl}
\hline Síntomas & Número y & porcentaje \\
\hline Disfagia & 15 & $(88,2 \%)$ \\
Disminución de peso & 11 & $(64,7 \%)$ \\
Epigastralgia & 10 & $(58,8 \%)$ \\
Vómito & 2 & $(11,7 \%)$ \\
Melenas & 2 & $(11,7 \%)$ \\
Hematemesis & 2 & $(11,7 \%)$ \\
Dolor retroesternal & 2 & $(11,7 \%)$ \\
Pirosis & 1 & $(5,8 \%)$
\end{tabular}


Sólo en ocho pacientes fue posible obtener datos sobre el estado nutricional previo, en quienes se encontró disminución leve en uno, moderada en dos y severa en tres. En los demás no hay datos suficientes que permitan hacer una evaluación objetiva de este parámetro.

Se realizaron 12 esofagectomías transhiatales y tres transtorácicas. El tipo de resección utilizado en enfermedad benigna fue transhiatal con interposición de colon en un paciente, y en otro resección de esófago torácico a través de una toracotomía posterolateral izquierda con esofagostomía, gastrostomía y yeyunostomía, por una perforación esofágica post-dilatación endoscópica. Para el enfermo con diagnóstico de acalasia se utilizó también la técnica transhiatal.

En todos los casos de enfermedad maligna se realizó un ascenso gástrico. La vía de reconstrucción en la esofagectomía transhiatal fue transmediastinal en diez y retroesternal en dos. En aquellos con esofagectomía transtorácica fue transmediastinal acompañado por disección de los ganglios mediastinales, con anastomosis cervical en dos de ellos e intratorácica en uno.

A un paciente con cáncer de la unión gastroesofágica se le practicó inicialmente una esofagogastrectomía total, pero no fue posible desde el punto de vista técnico realizarle interposición de colon ni de intestino delgado, por lo cual se realizó esofagostomía y yeyunostomía. Después del procedimiento, desarrolló como complicación una fístula de la yeyunostomía, lo que requirió lavados abdominales en varias oportunidades y por último falleció debido a sepsis de origen abdominal. La técnica quirúrgica de la esofagectomía transhiatal se hizo de acuerdo con la descrita por Orringer y la esofagectomía transtorácica según las descripciones de Mathissend del Ivor-Lewis y la de Pearson del McKeown (Tabla 3).

En 15 pacientes se realizó reconstrucción durante el procedimiento y en dos se practicó esofagostomía cervical. Para la reconstrucción en un segundo tiempo se realizaron 14 anastomosis cervicales y una intratorácica. Se utilizó técnica manual en 11 casos y sutura mecánica en cuatro. El tiempo quirúrgico varió entre tres y nueve horas, con un promedio de 6,1 horas.
El sangrado intraoperatorio estuvo entre $500 \mathrm{~mL}$ y $2500 \mathrm{~mL}$, siendo menor en la esofagectomía por enfermedad benigna. La mayoría osciló entre 500 y 1500 $\mathrm{mL}$, con un promedio de $1200 \mathrm{~mL}$.

La hospitalización duró entre diez y 48 días, con un promedio de 21 días. La estancia más corta correspondió al paciente a quien no se pudo practicar la reconstrucción y sufrió sepsis abdominal. El tiempo de estancia en la unidad de cuidado intensivo se encontró en el rango de uno a 20 días, para un promedio de cinco días. Uno de los pacientes no requirió manejo por esta unidad. El número de días en ventilación mecánica osciló entre uno (11 sujetos) y diez (un enfermo), con un promedio de 1,2 días.

Seis pacientes experimentaron complicaciones postoperatorias: fístula de la anastomosis esofagogástrica cervical, documentada mediante un trago de medio de contraste, en dos de ellos y fístula gástrica con la anastomosis indemne en un caso adicional. Otros eventos adversos fueron taquicardia ventricular (un caso), laringoespasmos, ${ }^{1}$ neumonía, ${ }^{1}$ sangrado postoperatorio proveniente de vasos tiroideos, demostrado por arteriografía, que requirió reintervención para ligadura, accidente cerebrovascular después de la arteriografía, edema pulmonar, ${ }^{1}$ mediastinitis ${ }^{2}$ y atonía gástrica. La presencia de neumotórax durante la disección transhiatal fue otra eventualidad, manejada con toracostomía bilateral.

Como complicaciones tardías se presentaron cinco estenosis cervicales de la anastomosis, demostradas por endoscopia digestiva alta, de las cuales cuatro fueron por esofagectomía transhiatal y una transtorácica. Uno de los individuos, operado por vía transtorácica, presentó fístula de la anastomosis cervical. Dos pacientes desarrollaron disfonía que fue estudiada con fibrolaringoscopia, demostrando parálisis unilateral de las cuerdas vocales, la cual casi siempre mejora meses después. Esta alteración explicada por lesión del nervio laríngeo recurrente, por lo regular asociada a esofagectomía transhiatal.

Tres pacientes fallecieron en el perioperatorio, uno por absceso intraabdominal y sepsis secundaria, otro por dehiscencia de la anastomosis esofagogástrica con ansures

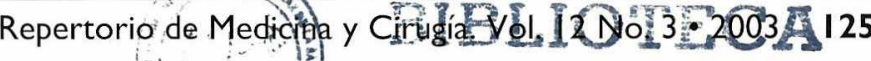




\begin{tabular}{|c|c|c|c|}
\hline Tipo de Cirugía & $\begin{array}{c}\text { Patología } \\
\text { maligna }\end{array}$ & $\begin{array}{c}\text { Patología } \\
\text { benigna }\end{array}$ & Total \\
\hline Transhiatal & 10 & 2 & 12 \\
\hline \multirow[t]{2}{*}{ Transtorácica } & 3 & 0 & 3 \\
\hline & & & 15 \\
\hline
\end{tabular}

Oringer donde los pacientes fueron extubados en la sala de recuperación y no necesitaron manejo en unidad de cuidado intensivo. En otras seis la estancia fue de 1,7 a 2,9 días. La duración de la ventilación mecánica fue de un día para 11 $(66 \%)$ de nuestros enfermos.

La mortalidad perioperatoria reportada en este estudio es del $23 \%$, mien-

mediastinitis y sepsis secundaria, y el tercero por una fístula gástrica al prácticar ascenso gástrico al cuello.

\section{Discusión}

Ninguno de los tres pacientes intervenidos por vía torácica fallecieron, los tiempos quirúrgicos fueron semejantes a los informados por Karl y sólo ocurrieron dos complicaciones. Los tiempos quirúrgicos permanecieron dentro del promedio reportado en otras series y no guardaron relación con la mortalidad perioperatoria.

En nuestro hospital existe una tendencia, o más bien preferencia, para la realización de esofagectomía trasnhiatal, sin discriminación tanto para enfermedad benigna como maligna, y sin razones aparentes o indicaciones específicas para ello.

El abordaje transtorácico a través de una incisión abdominal, toracotomía anterolateral derecha y cervicotomia, con anastomosis cervical esofagogástrica; tiene la ventaja de que no es necesario cambiar la posición del paciente durante el procedimiento y permite el trabajo simultáneo de dos equipos quirúrgicos, uno en el tórax y otro en el abdomen.

En nuestra serie la incidencia de fuga fue del $23 \%$ (tres pacientes), quienes fueron manejados con drenaje local cervical y suspensión de la vía oral. Uno de ellos desarrolló mediastanitis secundaria al absceso de la región cervical y falleció. La sutura mecánica se empleó en tres de nuestros pacientes, uno de los cuales presentó fístula.

El promedio de estancia en la unidad de cuidado intensivo fue cinco días, pero hay series como la de tras que otras series reportan una frecuencia de 5 a $8,1 \%$. En ellas también se informa una mayor incidencia de complicaciones pulmonares cuando se prefirió el procedimiento de Ivor - Lewis, al igual que una estancia prolongada tanto hospitalaria como en la unidad de cuidado intensivo. En nuestra serie la mortalidad se presentó en los pacientes a quienes se les realizó esofagectomía transhiatal. Sin embargo, estudios recientes de meta-análisis no han mostrado diferencias significativas en la mortalidad y complicaciones entre la técnica transhiatal y la transtorácica. Ninguno de los dos pacientes en quienes se utilizó la reconstrucción retroesternal, presentó mortalidad perioperatoria.

\section{Conclusiones}

Es infortunado que nuestro estudio no tuvo seguimiento alguno, de manera que resulta imposible la evaluación objetiva de los resultados a mediano y largo plazo de los ascensos gástricos y de la interposición del colon, en especial en los pacientes con enfermedad benigna.

Los resultados obtenidos no se pueden extrapolar a la población general, ya que la muestra obtenida fue muy pequeña, por lo cual consideramos necesarias nuevas investigaciones con una población mayor.

\section{Lecturas recomendadas}

1. Bartels H, Stein H, Siewert J. Preoperative risk analysis and postoperative mortality of oesophagectomy for respectable oesophageal cancer. Br J Surg 1998; 85: 840-44

2. Collard JM, Otte JB, Fiasse R, et al. Skeletonizing on bloc esophagectomy for cancer. Ann Surg 2001; 234:25-32

3. Collard JM. Exclusive radical surgery for esophageal adenocarcinoma. Cancer 2001; 91:1098-1104.

4. Gawad K, Hosch S, Bumann D, et al. How important is the 
route of reconstruction after esophagectomy: a prospective randomized study. Am J of Gastroenterol 1999;94:1490 1495.

5. Gluch L, Smith R, Banbach C, et al. Comparison of outcomes following transhiatal or Ivor Lewis esophagectomy for esophageal carcinoma. World J Surg 1999; 23: 271 - 76.

6. Griffin $S$, Lamb P, Dresner S, et al. Diagnosis and management of a mediastinal leak following radical esophagectomy. Br J Surg 2001; 88:1346-51

7. Hulscher J, Van Sandick J, Tijssen J, et al. The recurrence pattern of esophageal carcinoma after transhiatal resection. J Am Coll Surg 2000; 191:143-48

8. Hulscher J, Tijssen J, Obertop H, et al. Transthoracic versus transhiatal resection for carcinoma of the esophagus: A meta-analysis. Ann Thorac Surg 2001; 72: 306 - 13

9. Karl R, Schreiber R, Boulware D, et al. Factors affecting morbidity, mortality and survival in patients undergoing Ivor Lewis esophagogastrectomy. Ann Surg 2000; 231: 635 $-43$.

10. Kim S, Lee K, Shim Y, et al. Esophageal resection: indications, techniques and radiologic assessment. Radiographics 2001; 21:1119-1140.

11. Law S, Wong J. What is appropriate treatment for carcinoma of the thoracic esophagus? World J Surg 2001; 25:189- 191.
12. Lee R, Miller J. Esofagectomía por cáncer: Clínicas Quirúrgicas de Norteamérica 1997; 5:1141 - 1165

13. Orringer M, Marshall B, Lannettoni M. Transhiatal esophagectomy for treatment of benign and malignant esophageal disease. World J Surg 2001; 25: 196 - 203.

14. Orringer M, Marshall B, Lannettoni M. Transhiatal esophagectomy: clinical experience and refinements. Ann Surg 1999; 230: $392-403$.

15. Zuidema G. Techniques of esophageal reconstruction. Surgery of the alimentary tract. W. B. Saunders Eds. 1996: $389-445$.

16. Singh D, Maley R, Santucci T, et al. Experience and technique of stapled mechanical cervical esophagogastric anastomosis. Ann Thorac Surg 2001; 71:419-24

17. Stone C, Heitmiller R. Simplified, standarized technique for cervical esophagogastric anastomosis. Ann Thorac Surg 1994; 58: $259-61$

18. Visbal A, Allen M, Miller D. Ivor Lewis esophagogastrectomy for esophageal cancer. Ann Thorac Surg 2001; 71:1803-8.

19. Zinner M. Procedimientos quirúrgicos para resecar y reemplazar el esófago. Operaciones abdominales. Ed. Médica Panamericana 1998: 821 - 844

20. Pearson F. Esophageal surgery. Churchill Livingstone ed. 1995: 669-682.

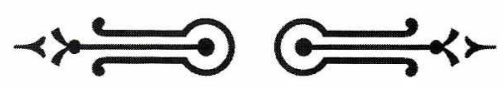

